\title{
Extraction Optimization for Obtaining Artemisia capillaris Extract with High Anti-Inflammatory Activity in RAW 264.7 Macrophage Cells
}

\author{
Mi Jang, ${ }^{1,2}$ Seung-Weon Jeong, ${ }^{1}$ Bum-Keun Kim, ${ }^{1}$ and Jong-Chan Kim ${ }^{1}$ \\ ${ }^{1}$ Korea Food Research Institute, 1201-62 Anyangpangyo-ro, Bundang-gu, Seongnam-si, Gyeonggi-do 463-746, Republic of Korea \\ ${ }^{2}$ Department of Oriental Medicinal Material and Processing, College of Life Science, Kyung Hee University, 1732 Deogyeong-daero, \\ Giheung-gu, Yongin-si, Gyeonggi-do 446-701, Republic of Korea
}

Correspondence should be addressed to Jong-Chan Kim; jckim@kfri.re.kr

Received 16 February 2015; Revised 24 April 2015; Accepted 28 April 2015

Academic Editor: Ruxana Sadikot

Copyright (C) 2015 Mi Jang et al. This is an open access article distributed under the Creative Commons Attribution License, which permits unrestricted use, distribution, and reproduction in any medium, provided the original work is properly cited.

Plant extracts have been used as herbal medicines to treat a wide variety of human diseases. We used response surface methodology (RSM) to optimize the Artemisia capillaris Thunb. extraction parameters (extraction temperature, extraction time, and ethanol concentration) for obtaining an extract with high anti-inflammatory activity at the cellular level. The optimum ranges for the extraction parameters were predicted by superimposing 4-dimensional response surface plots of the lipopolysaccharide- (LPS-) induced $\mathrm{PGE}_{2}$ and NO production and by cytotoxicity of $A$. capillaris Thunb. extracts. The ranges of extraction conditions used for determining the optimal conditions were extraction temperatures of $57-65^{\circ} \mathrm{C}$, ethanol concentrations of $45-57 \%$, and extraction times of 5.5-6.8 h. On the basis of the results, a model with a central composite design was considered to be accurate and reliable for predicting the anti-inflammation activity of extracts at the cellular level. These approaches can provide a logical starting point for developing novel anti-inflammatory substances from natural products and will be helpful for the full utilization of $A$. capillaris Thunb. The crude extract obtained can be used in some A. capillaris Thunb.-related health care products.

\section{Introduction}

Plant extracts have been used as herbal medicines to treat a wide variety of human diseases. The herbal products today symbolize safety in contrast to synthetics, which are regarded as unsafe to humans and the environment [1]. The use of herbal and natural products in East Asian countries is increasing because of their pharmacological or biological activities [2]. Among the numerous herbs used in Oriental medicine, Artemisia capillaris Thunb. is one of the earliest and most important edible crude herbs used for medicinal purposes in Korea, China, and Japan. A. capillaris has been widely used as a hepatoprotective, analgesic, and antipyretic agent [3]. Many researchers have studied its various biological activities, such as anti-inflammatory [4], antioxidant [5], anticarcinogenic [6], and antimicrobial [3] properties.

Inflammation is a multistep process mediated by activated inflammatory and immune cells, including macrophages and monocytes [7], and comprises a complex series of reactions regulated by a cascade of cytokines, growth factors, nitric oxide (NO), and prostaglandins (PGs) produced by active macrophages [8]. Inflammation is one of the most important defense mechanisms, but prolonged inflammation contributes to the pathogenesis of many inflammatory diseases, including bronchitis [9], gastritis [10], inflammatory bowel disease [11], multiple sclerosis [12], and rheumatoid arthritis [13]. The employment of a variety of anti-inflammatory agents may help in the therapeutic treatment of pathologies associated with inflammation. The development and utilization of more effective anti-inflammatory agents of natural origin are therefore required.

Extraction is the first critical stage in the preparation of plant formulations. The crude extracts directly obtained from plants can be used as a remedial agent or the crude part can be further fractionated and purified by chemicals and solvents. Overall, the crude extracts finally lead to 
herbal drugs, which all have traditional medicinal value. Therefore, the standardization of extracts and extraction methods are important in the field of phytochemistry [14]. Modern methods of extraction are effective in advancing the development of traditional herbal remedies [15]. Response surface methodology (RSM) has been widely used to optimize extraction conditions such as temperature, extraction time, and concentration of solvents. RSM consists of mathematical and statistical techniques used to develop an adequate functional relationship between a response of interest and some independent variable [16].

With the increasing demand for herbal medicinal products and natural products for health care all over the world, herbal manufacturers aim for the most appropriate extraction technologies to produce extracts of defined quality with the lowest batch-to-batch variation, which can also help in the scaling-up of extraction. To have a complete understanding of the bioactivity of crude extracts, it is necessary to optimize the extraction methodology to achieve the broadest possible range of phytochemicals [17]. The objective of the present study was to apply the RSM approach to optimize the extraction temperature, extraction time, and ethanol concentration to maximize the anti-inflammatory activities from A. capillaris Thunb. at the cellular level. The crude extract obtained can be used in some A. capillaris Thunb.related health care products. Thus, the results obtained will be helpful for the full utilization of $A$. capillaris Thunb.

\section{Materials and Methods}

2.1. Plant Materials and Extract Preparation. In March 2013, whole plants of $A$. capillaris Thunb. were obtained from the Department of Oriental Pharmacy, Kyung Hee Medical Center, Seoul, Korea. Voucher specimens of the plant materials are kept in our laboratory (Korea Food Research Institute, Gyeonggi, Korea) for further reference. The dried sample was ground in a blender to obtain a fine powder (particle diameter size: $500-850 \mu \mathrm{m}$ ). Five grams of dried A. capillaris Thunb. powder was extracted by $100 \mathrm{~mL}$ of different ethanol concentrations at the required temperature and duration. Each extract was filtered using filter paper (Whatman number 4). The ethanol was removed under reduced pressure by rotary evaporation, and the water residue was removed by lyophilization. For testing, the extracts were dissolved in phosphate-buffered saline and diluted to the desired concentrations.

2.2. Cell Culture. RAW 264.7 macrophages were obtained from the Korean Cell Line Bank (KCLB, Seoul, Korea) and were maintained in RPMI 1640 medium (Gibco-BRL, Grand Island, NY, USA) containing antibiotics (100 units/mL penicillin $\mathrm{A}$ and $100 \mu \mathrm{g} / \mathrm{mL}$ streptomycin) and $10 \%$ heatinactivated fetal bovine serum (Gibco-BRL, USA) at $37^{\circ} \mathrm{C}$ in a $5 \% \mathrm{CO}_{2}$ incubator.

2.3. MTT Assay. The viability of the cells was determined colorimetrically by using the MTT assay as described by Denizot and Lang [18] with some modification. The RAW
264.7 cells were seeded in a 96-well plate at a density of $5 \times$ $10^{5}$ cell $\mathrm{mL}^{-1}$ and were treated with various concentrations of the extracts for $24 \mathrm{~h}$. MTT solution $(20 \mu \mathrm{L}$ of $5 \mathrm{mg} / \mathrm{mL}$ MTT in phosphate-buffered saline) was added to each well, and the cells were incubated for $2 \mathrm{~h}$. After the supernatants were aspirated, the formazan crystals in each well were dissolved in $100 \mu \mathrm{L}$ of dimethyl sulfoxide (DMSO) and the optical density (OD) of cells at $570 \mathrm{~nm}$ was measured using a microplate reader (Bio-Rad, Hercules, CA, USA). The OD of the samples was compared to that of the LPS-untreated control to obtain the percentage viability.

2.4. Anti-Inflammatory Activity. The anti-inflammatory activities of the extracts were measured using the $\mathrm{NO}$ and $\mathrm{PGE}_{2}$ assays. Both assays have been widely used to determine the anti-inflammatory activity of medicinal plants. These methods were selected because they are based on different principles and because they are widely used. NO is a wellknown proinflammatory mediator that is involved in various physiological and pathological processes. Recently, the suppression of NO production has been emphasized as a new pharmacological strategy for the treatment of inflammationrelated diseases [19]. $\mathrm{PGE}_{2}$ is a key inflammatory mediator and stimulates cytokine generation and vasodilation and mediates fever and pain [20]. These two mediators are believed to be adequate to assess the anti-inflammatory activity of $A$. capillaris Thunb. extract.

2.5. Measurement of NO Production. The NO level in the cultured medium was determined by the Griess reaction [21]. The cells were pretreated with the indicated concentrations of the extracts for $2 \mathrm{~h}$ and then were induced with a $1 \mu \mathrm{g} / \mathrm{mL}$ concentration of LPS for an additional $22 \mathrm{~h}$. Supernatant from each well $(100 \mu \mathrm{L})$ was mixed with $100 \mu \mathrm{L}$ of Griess reagent in a separate 96 -well plate. After incubation for $15 \mathrm{~min}$ at room temperature, the OD was measured at $540 \mathrm{~nm}$ using a microplate reader. NO production by the extract-treated cells is presented as a percentage of the NO production in the LPSinduced control.

2.6. Measurement of $P G E_{2}$ Production. RAW 264.7 cells were incubated with LPS $\left(1 \mu \mathrm{g} \mathrm{mL}^{-1}\right)$ in the presence or absence of the samples for $24 \mathrm{~h}$. The samples were analyzed using $\mathrm{PGE}_{2}$ enzyme immune assay (EIA) kit (R\&D Systems, Minneapolis, $\mathrm{MN}$ ), according to the manufacturer's protocol. $\mathrm{PGE}_{2}$ concentrations in the supernatants were determined by comparison with a standard curve. $\mathrm{PGE}_{2}$ production by the extract-treated cells is presented as the percentage of $\mathrm{PGE}_{2}$ production in the LPS-induced control.

2.7. Experimental Design. The RSM was used to optimize extraction conditions and monitor the extraction characteristics. The experimental design was a central composite design (CCD). The 3 independent variables were extraction temperature $\left(X_{1}\right)$, ethanol concentration $\left(X_{2}\right)$, and extraction time $\left(X_{3}\right)$, and the response variables were LPS-induced $\operatorname{PGE}_{2}\left(Y_{1}\right)$ and NO $\left(Y_{2}\right)$ production and cytotoxicity $\left(Y_{3}\right)$ in RAW 264.7 cells. Each independent variable to be optimized 
TABle 1: The central composite experimental design and experimental data for the optimization of conditions for obtaining Artemisia capillaris Thunb. extracts.

\begin{tabular}{|c|c|c|c|c|c|c|}
\hline \multirow{2}{*}{ Run } & \multicolumn{3}{|c|}{ Independent variables ${ }^{1}$} & \multicolumn{3}{|c|}{ Response variables $^{2}$} \\
\hline & $X_{1}\left({ }^{\circ} \mathrm{C}\right)$ & $X_{2}(\%)$ & $X_{3}(\mathrm{~h})$ & $Y_{1}(\%)$ & $Y_{2}(\%)$ & $Y_{3}(\%)$ \\
\hline 1 & $42(-1)$ & $20(-1)$ & $3(-1)$ & $73.38 \pm 0.21$ & $79.18 \pm 1.19$ & $87.34 \pm 2.04$ \\
\hline 2 & $78(1)$ & $20(-1)$ & $3(-1)$ & $78.81 \pm 0.15$ & $83.32 \pm 0.24$ & $84.98 \pm 4.38$ \\
\hline 3 & $42(-1)$ & $80(1)$ & $3(-1)$ & $86.69 \pm 0.13$ & $87.21 \pm 0.33$ & $80.36 \pm 0.52$ \\
\hline 4 & $78(1)$ & $80(1)$ & $3(-1)$ & $67.11 \pm 0.23$ & $69.34 \pm 0.32$ & $81.46 \pm 0.89$ \\
\hline 5 & $42(-1)$ & $20(-1)$ & $9(1)$ & $74.38 \pm 0.58$ & $69.55 \pm 1.06$ & $85.98 \pm 1.19$ \\
\hline 6 & $78(1)$ & $20(-1)$ & $9(1)$ & $85.69 \pm 0.79$ & $85.08 \pm 0.88$ & $82.56 \pm 1.34$ \\
\hline 7 & $42(-1)$ & $80(1)$ & $9(1)$ & $68.32 \pm 0.25$ & $69.50 \pm 0.24$ & $82.88 \pm 1.34$ \\
\hline 8 & $78(1)$ & $80(1)$ & $9(1)$ & $73.38 \pm 0.14$ & $72.50 \pm 0.16$ & $86.92 \pm 2.15$ \\
\hline 9 & $30(-1.682)$ & $50(0)$ & $6(0)$ & $75.15 \pm 0.42$ & $78.66 \pm 0.24$ & $89.89 \pm 0.41$ \\
\hline 10 & $90(1.682)$ & $50(0)$ & $6(0)$ & $63.41 \pm 0.11$ & $63.18 \pm 0.31$ & $88.13 \pm 2.75$ \\
\hline 11 & $60(0)$ & $0(-1.682)$ & $6(0)$ & $71.65 \pm 0.52$ & $80.68 \pm 0.24$ & $85.24 \pm 0.37$ \\
\hline 12 & $60(0)$ & $100(1.682)$ & $6(0)$ & $75.15 \pm 0.81$ & $83.84 \pm 0.16$ & $85.50 \pm 1.41$ \\
\hline 13 & $60(0)$ & $50(0)$ & $1(-1.682)$ & $69.01 \pm 0.19$ & $75.92 \pm 0.27$ & $80.28 \pm 1.30$ \\
\hline 14 & $60(0)$ & $50(0)$ & 11 (1.682) & $66.71 \pm 0.14$ & $71.36 \pm 0.32$ & $88.00 \pm 0.26$ \\
\hline 15 & $60(0)$ & $50(0)$ & $6(0)$ & $53.21 \pm 0.21$ & $56.50 \pm 0.98$ & $90.47 \pm 1.26$ \\
\hline 16 & $60(0)$ & $50(0)$ & $6(0)$ & $54.14 \pm 0.47$ & $56.03 \pm 1.79$ & $91.20 \pm 1.15$ \\
\hline 17 & $60(0)$ & $50(0)$ & $6(0)$ & $54.36 \pm 0.23$ & $56.03 \pm 0.86$ & $90.75 \pm 1.89$ \\
\hline 18 & $60(0)$ & $50(0)$ & $6(0)$ & $54.75 \pm 0.15$ & $57.12 \pm 0.28$ & $90.13 \pm 0.74$ \\
\hline 19 & $60(0)$ & $50(0)$ & $6(0)$ & $53.97 \pm 0.50$ & $56.65 \pm 0.91$ & $91.75 \pm 1.71$ \\
\hline 20 & $60(0)$ & $50(0)$ & $6(0)$ & $54.36 \pm 0.14$ & $55.87 \pm 1.07$ & $90.13 \pm 2.34$ \\
\hline
\end{tabular}

${ }^{1}$ Independent variables: $X_{1}$, extraction temperature; $X_{2}$, ethanol concentration; $X_{3}$, extraction time.

${ }^{2}$ Response variables: $Y_{1}$, LPS-induced $\mathrm{PGE}_{2}$ production; $Y_{2}$, LPS-induced NO production; $Y_{3}$, cytotoxicity.

was coded at 5 levels $(-1.682,-1,0,1$, and 1.682) with 20 runs, including 6 replicates at the central point (Table 1). Experimental data were analyzed using the Statistical Analysis System (SAS) program (SAS Inst. Inc., Cary, NC, USA) and fitted to a second-order polynomial regression model containing the coefficient of linear, quadratic, and two-factor interaction effects. The model equation for the response of the 3 independent variables was

$$
\begin{aligned}
Y_{n}= & \beta_{0}+\beta_{1} X_{1}+\beta_{2} X_{2}+\beta_{3} X_{3}+\beta_{11} X_{1}^{2}+\beta_{22} X_{2}^{2} \\
& +\beta_{33} X_{3}^{2}+\beta_{1} \beta_{2} X_{1} X_{2}+\beta_{1} \beta_{3} X_{1} X_{3} \\
& +\beta_{2} \beta_{3} X_{2} X_{3} .
\end{aligned}
$$

In this model, $Y_{n}$ is the predicted response variable; $\beta_{0}$ is the constant coefficient; $\beta_{1}, \beta_{2}$, and $\beta_{3}$ are the regression coefficients for the linear effect terms; $\beta_{11}, \beta_{22}$, and $\beta_{33}$ are the quadratic effect terms; and $\beta_{1} \beta_{2}, \beta_{1} \beta_{3}$, and $\beta_{2} \beta_{3}$ are the interaction effect terms. The adequacy of the model was predicted through regression analysis $\left(R^{2}\right.$ and adjusted $\left.R^{2}\right)$ and analysis of variance (ANOVA) $(P<0.05)$. The relationship between the independent variables $\left(X_{1}, X_{2}\right.$, and $\left.X_{3}\right)$ and the response variables $\left(Y_{1}, Y_{2}\right.$, and $\left.Y_{3}\right)$ was demonstrated through four-dimensional response surface plots generated using the Wolfram Mathematica software (Wolfram Research, Inc., Champaign, Illinois, USA) [22].

2.8. Prediction and Verification of Optimum Extraction Conditions. The optimum ranges of the extraction conditions were predicted by superimposing the response surfaces of the LPS-induced $\mathrm{PGE}_{2}$ and $\mathrm{NO}$ production, which are the representative inflammatory factors, and by cytotoxicity in vitro. The optimum extraction conditions were verified by comparing the experimental values with the predicted values.

2.9. HPLC Analysis. The analytical HPLC system employed consisted of a JASCO high-performance liquid chromatograph coupled with a UV-Vis multiwavelength detector (MD910 JASCO). HPLC analysis operated under the following conditions: YMC-Pack ODS-AM column $(250 \mathrm{~mm} \times 4.6 \mathrm{~mm}$ i.d. and particles of $5 \mu \mathrm{m}$ ) (YMC, Japan), column oven temperature $35^{\circ} \mathrm{C}$, and detection $285 \mathrm{~nm}$. The gradient solvent system consisted of $0.1 \%$ acetic acid in water (solvent $\mathrm{A}$ ) and $0.1 \%$ acetic acid in acetonitrile/water (solvent $\mathrm{B}$ ) as follows: 0 $1 \mathrm{~min}, 12 \% \mathrm{~B}$; $1-18 \mathrm{~min}, 22 \% \mathrm{~B}$; $18-28 \mathrm{~min}, 28 \% \mathrm{~B}$; $28-35 \mathrm{~min}$, $38 \% \mathrm{~B}$; $35-48 \mathrm{~min}, 48 \% \mathrm{~B}$; $48-54 \mathrm{~min}, 68 \% \mathrm{~B}$; 54-60 min, $100 \% \mathrm{~B} ; 60-67 \mathrm{~min}, 12 \%$. The flow rate was $1.0 \mathrm{~mL} \mathrm{~min}^{-1}$ and the injection volume was $10 \mu \mathrm{L}$. The identification of each compound was based on a combination of retention time and spectral matching.

\section{Results and Discussion}

The effects of three independent process variables-extraction temperature $\left(X_{1}, 30-90^{\circ} \mathrm{C}\right)$, ethanol concentration $\left(X_{2}\right.$, $0-100 \%)$, and extraction time $\left(X_{3}, 1-11 \mathrm{~h}\right)$-were investigated and CCD was applied to determine the optimal combination 
TABLE 2: Regression coefficients of the predicted second-order polynomial models and the results of an analysis of variance for LPS-induced $\mathrm{PGE}_{2}$ and NO production and cytotoxicity in RAW 264.7 cells.

\begin{tabular}{|c|c|c|c|c|c|c|c|c|c|}
\hline \multirow{2}{*}{ Source } & \multicolumn{3}{|c|}{ LPS-induced $\mathrm{PGE}_{2}$ production } & \multicolumn{3}{|c|}{ LPS-induced NO production } & \multicolumn{3}{|c|}{ Cytotoxicity } \\
\hline & Coefficients & $F$-value & $P$ value & Coefficients & $F$-value & $P$ value & Coefficients & $F$-value & $P$ value \\
\hline$\overline{\beta_{0}}$ & 173.194583 & 8.42 & $<0.0001$ & 175.977805 & 9.90 & $<0.0001$ & 72.885069 & 9.10 & $<0.0001$ \\
\hline \multicolumn{10}{|l|}{ Linear } \\
\hline$\beta_{1}$ & -2.523157 & -5.11 & 0.0005 & -2.059834 & -4.82 & 0.0007 & 0.320093 & 1.66 & 0.1274 \\
\hline$\beta_{2}$ & -0.289006 & -1.19 & 0.2625 & -0.528652 & -2.51 & 0.0308 & -0.037478 & -0.40 & 0.7010 \\
\hline$\beta_{3}$ & -11.023825 & -4.38 & 0.0014 & -12.992655 & -5.97 & 0.0001 & 3.116604 & 3.18 & 0.0099 \\
\hline \multicolumn{10}{|l|}{ Quadratic } \\
\hline$\beta_{11}$ & 0.019932 & 5.50 & 0.0003 & 0.016046 & 5.12 & 0.0005 & -0.004113 & -2.91 & 0.0155 \\
\hline$\beta_{22}$ & 0.008825 & 6.76 & $<0.0001$ & 0.010313 & 9.14 & $<0.0001$ & -0.002561 & -5.04 & 0.0005 \\
\hline$\beta_{33}$ & 0.660889 & 5.06 & 0.0005 & 0.686408 & 6.08 & 0.0001 & -0.306064 & -6.02 & 0.0001 \\
\hline \multicolumn{10}{|l|}{ Interaction } \\
\hline$\beta_{1} \beta_{2}$ & -0.007467 & -2.59 & 0.0268 & -0.007997 & -3.21 & 0.0093 & 0.002755 & 2.45 & 0.0340 \\
\hline$\beta_{1} \beta_{3}$ & 0.072940 & 2.53 & 0.0298 & 0.074690 & 3.00 & 0.0133 & 0.002083 & 0.19 & 0.8564 \\
\hline$\beta_{2} \beta_{3}$ & -0.029131 & -1.69 & 0.1228 & -0.009278 & -0.62 & 0.548 & 0.017361 & 2.58 & 0.0275 \\
\hline $\operatorname{SSPE}^{1}$ & 1.366898 & & & 1.129981 & & & 1.635000 & & \\
\hline Total model & & 11.56 & 0.0003 & & 17.10 & $<0.0001$ & & 16.97 & 0.0037 \\
\hline$R^{2}$ & 0.9123 & & & 0.9390 & & & 0.8941 & & \\
\hline Adjusted $R^{2}$ & 0.8334 & & & 0.8841 & & & 0.7988 & & \\
\hline
\end{tabular}

${ }^{1}$ Pure error of the sum of squares.

of the factors. The three responses of interest were LPSinduced $\mathrm{PGE}_{2}$, NO production, and cytotoxicity. The results of 20 runs by using the CCD design are shown in Table 1, which include the coded matrices for design conditions and corresponding results of RSM experiments.

3.1. Cell Viability. We examined the cytotoxic effects of $A$. capillaris Thunb. extracts on RAW 264.7 cells and found that none of the extracts affected cell viability at 5, 10, or $30 \mu \mathrm{g} / \mathrm{mL}$ after $24 \mathrm{~h}$. However, the extracts inhibited cell viability at $50 \mu \mathrm{g} / \mathrm{mL}$ (data not shown). Thus, a concentration of $30 \mu \mathrm{g} / \mathrm{mL}$ was used to treat the cells in the following experiments.

3.2. Optimization of LPS-Induced PGE 2 Production. $\mathrm{PGE}_{2}$ is a mediator of active inflammation and a bioactive lipid that can elicit a wide range of biological effects associated with inflammation and cancer. It plays a critical role in guiding and governing various aspects of the inflammatory response. The role of $\mathrm{PGE}_{2}$ in driving acute inflammation is well established [23].

3.3. Model Fitting and Statistical Analysis. Table 2 shows the results of fitting quadratic models to the data. The results of ANOVA indicate that the contribution of the quadratic model was significant. A quadratic regression model for LPSinduced $\mathrm{PGE}_{2}$ production was obtained from the experimental data, as shown by using

$$
\begin{aligned}
\mathrm{PGE}_{2}= & 173.194583-2.523157 X_{1}-0.289006 X_{2} \\
& -11.023825 X_{3}+0.019932 X_{1}^{2}
\end{aligned}
$$

$$
\begin{aligned}
& +0.008825 X_{2}^{2}+0.660889 X_{3}^{2} \\
& -0.007467 X_{1} X_{2}+0.072940 X_{1} X_{3} \\
& -0.029131 X_{2} X_{3}
\end{aligned}
$$

An ANOVA was performed to check the adequacy of the suggested models and identify the significant factors; a statistical summary is given in Table 2. The model Fvalue of 11.56 for LPS-induced $\mathrm{PGE}_{2}$ production implies that the model is statistically significant. There was only a $0.03 \%$ chance that a model $F$-value this large could occur randomly. The $P$ value of less than 0.05 indicates that the model terms were also significant. Furthermore, the value of pure error was low, which indicates good reproducibility of the obtained data, with a low $P$ value from the ANOVA and a satisfactory coefficient of determination (Table 2). The total determination coefficient $\left(R^{2}\right)$ was 0.9123 , which indicates that $91.23 \%$ of the variability in the response variables was explained and only $8.77 \%$ of the total variation was not explained using the model. The $R^{2}$ indicates the observed variability in the data that was accounted for by using the model. The adjusted $R^{2}$ (adj. $R^{2}$ ) modifies the $R^{2}$ by taking into account the number of covariates or predictors in the model [24]. The adj. $R^{2}$ was 0.8334 , which suggests that there are excellent correlations between the independent variables. Several studies have supported the acceptance of any model with an $R^{2}$ value $>0.75[25,26]$. The significance of each coefficient, which was determined using the $F$-test, and $P$ values are shown in Table 2 . The corresponding variables would have been more significant if the absolute $F$-value was greater and the $P$ value was smaller [27]. Table 2 shows 




(a)

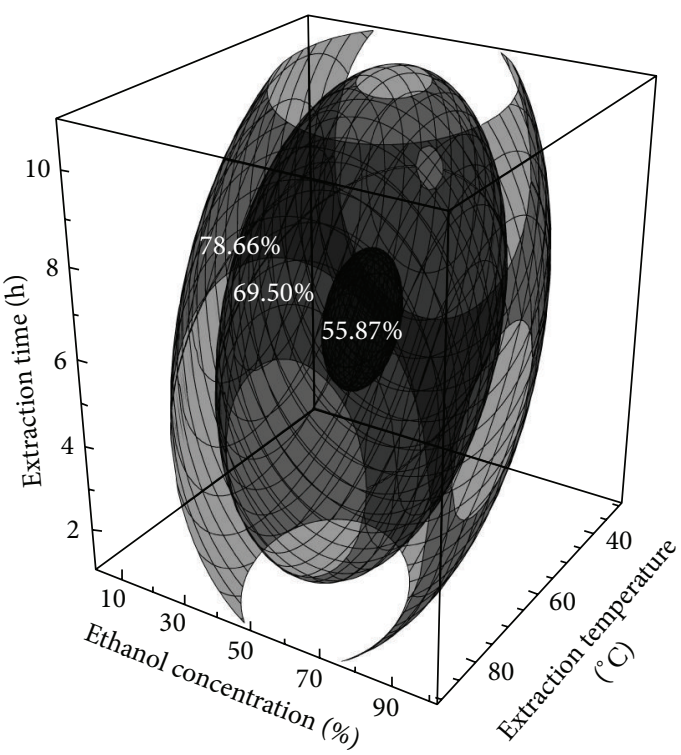

(b)

FIGURE 1: The 4-dimensional response surface plots showing the combined effect of extraction temperature, ethanol concentration, and extraction time on LPS-induced $\mathrm{PGE}_{2}$ (a) and NO production (b).

that the linear terms $X_{1}$ and $X_{3}$, all quadratic terms, and interaction terms $X_{1} X_{2}$ and $X_{1} X_{3}$ had significant effects $(P<0.001$ or $P<0.05)$, whereas the linear term $X_{2}$ and interaction term $X_{2} X_{3}$ did not have significant effects on LPS-induced $\mathrm{PGE}_{2}$ production $(P>0.05)$.

3.4. Canonical Analysis of the Stationary Point. Canonical analysis is a mathematical approach used to locate the stationary point of the response surface in the experimental region and to determine whether it represents a maximum, minimum, or saddle point [28]. The canonical form of the fitted LPS-induced $\mathrm{PGE}_{2}$ production is shown by using

$$
\begin{aligned}
Y_{1}= & 53.826529+29.048986 \omega_{1}{ }^{2}+15.900304 \omega_{2}{ }^{2} \\
& +11.573887 \omega_{3}{ }^{2}
\end{aligned}
$$

where $\omega_{1}, \omega_{2}$, and $\omega_{3}$ denote the transformed independent variables or the canonical variables. All the eigenvalues from (3) are positive, which indicates a unique minimum LPSinduced $\mathrm{PGE}_{2}$ production at the stationary point.

In the graphical approach, the predictive model for the LPS-induced $\mathrm{PGE}_{2}$ production was modified and used to create the four-dimensional response surface within the experimental region using a Mathematica program. As shown in Figure 1(a), the LPS-induced $\mathrm{PGE}_{2}$ production decreased as the extraction temperature and extraction time minimally increased (extraction temperature, $62^{\circ} \mathrm{C}$; extraction time, $6 \mathrm{~h}$; and ethanol concentration, 53\%); therefore, the predicted stationary point was at the minimum (53.8\%). The LPSinduced $\mathrm{PGE}_{2}$ production increased as the extraction temperature, extraction time, and ethanol concentration were increased above the minimum values. The ethanol extract of A. capillaris has been shown to have high levels of antioxidant activity [29] and to contain several other bioactive flavonoids, including scoparone, capillarisin, cirsimaritin, genkwanin, and rhamnocitrin [30]. It has been reported that the ethanol extract of $A$. capillaris exerted an anti-inflammatory effect on the mRNA expression level of cyclooxygenase-2 (COX2) in LPS-stimulated RAW 264.7 cells [31]. COX-2 is an enzyme that generates prostaglandins, which are induced by proinflammatory cytokines and other activators, such as LPS, resulting in the release of a large amount of $\mathrm{PGE}_{2}$ at inflammation sites [32]. $\mathrm{PGE}_{2}$ is a major inflammatory lipid mediator involved in the pathogenesis of chronic inflammatory diseases such as rheumatoid arthritis, and it is synthesized by macrophages and other cell types in the presence of LPS $[33,34]$. These results suggest that the extraction conditions might influence the decrease in $\mathrm{PGE}_{2}$ production in LPS-induced RAW 264.7 cells and provide a crude extract with high anti-inflammatory activity. The beneficial effects of nonsteroidal anti-inflammatory drugs (NSAID) in the treatment of inflammatory diseases have been well documented in the last decades. Evidence exists that these effects are mediated by an effective limitation of the production of $\mathrm{PGE}_{2}$ at the site of inflammation [35].

3.5. Optimization of LPS-Induced NO Production. Recently, suppression of the level of NO generation has been emphasized as a new pharmacological strategy for the treatment of inflammation-related diseases [19]. NO production may reflect the degree of inflammation and provides a measure to assess the effect of chemopreventive agents on the inflammatory process.

3.6. Model Fitting and Statistical Analysis. On the basis of the experimental results of CCD (Table 1) and regression 
analysis, an equation for the response surface was developed to estimate the relationship between the LPS-induced NO production and the independent variables $\left(X_{1}, X_{2}\right.$, and $\left.X_{3}\right)$. The model could be expressed as follows:

$$
\begin{aligned}
\mathrm{NO}= & 175.977805-2.059834 X_{1}-0.528652 X_{2} \\
& -12.992655 X_{3}+0.016046 X_{1}^{2}+0.010313 X_{2}^{2} \\
& +0.686408 X_{3}^{2}-0.007997 X_{1} X_{2} \\
& +0.074690 X_{1} X_{3}-0.009278 X_{2} X_{3} .
\end{aligned}
$$

The results of the ANOVA for the adequacy and fitness of the models are summarized in Table 2. The data indicated that the proposed regression model for the LPS-induced NO production was adequate with a satisfactory $R^{2}$ value (determined coefficient). The $R^{2}$ value for the LPS-induced NO production was 0.9390 , which showed a close agreement between the experimental results and the theoretical values predicted by the polynomial model. These results, along with the high model $F$-value of 17.10 , imply that the predicted model for the LPS-induced NO production was significant $(P<0.0001)$ and adequate. The $P$ values are used as a tool to check the significance of each coefficient. The smaller the magnitude of the $P$ value is the more significant the corresponding coefficient is, and this strongly affects the response variable [36]. From Table 2, the quadratic term of the ethanol concentration $\left(X_{2}{ }^{2}\right)$ had the largest effect on LPSinduced NO production, as indicated by its lowest $P$ value $(<0.0001)$ and highest absolute $F$-value (9.14). Next, the linear terms of the extraction temperature $\left(X_{1}\right)$ and extraction time $\left(X_{3}\right)$ show a substantial significant effect at a $99.9 \%$ confidence level $(P<0.001)$, and the interaction term $X_{1} X_{2}$ were significant $(P<0.01)$.

3.7. Canonical Analysis of the Stationary Point. The canonical analysis revealed that the stationary point was a minimum and the canonical form of the fitted response model could be depicted by the equation

$$
\begin{aligned}
Y_{2}= & 56.069620+29.351142 \omega_{1}{ }^{2}+19.055285 \omega_{2}{ }^{2} \\
& +8.978654 \omega_{3}{ }^{2} .
\end{aligned}
$$

As the eigenvalues were all positive, the stationary point was a minimum. On the basis of the predicted model, a four-dimensional response surface for LPS-induced NO production is shown in Figure 1(b). LPS-induced NO production had a minimum predicted value at $56 \%$, obtained under the following conditions: $62^{\circ} \mathrm{C}$ extraction temperature, $52 \%$ ethanol concentration, and $6 \mathrm{~h}$ extraction time. On the basis of the results shown in Figure 1(b), at low extraction temperatures and a short extraction time, the LPSinduced NO production first decreased and then increased with increasing ethanol concentration, suggesting that an intermediate ethanol concentration is favorable. NO is the key regulator of immune responses and is involved in various physiological and pathological processes. Therefore, NO is a potential target for new therapeutic strategies, and the

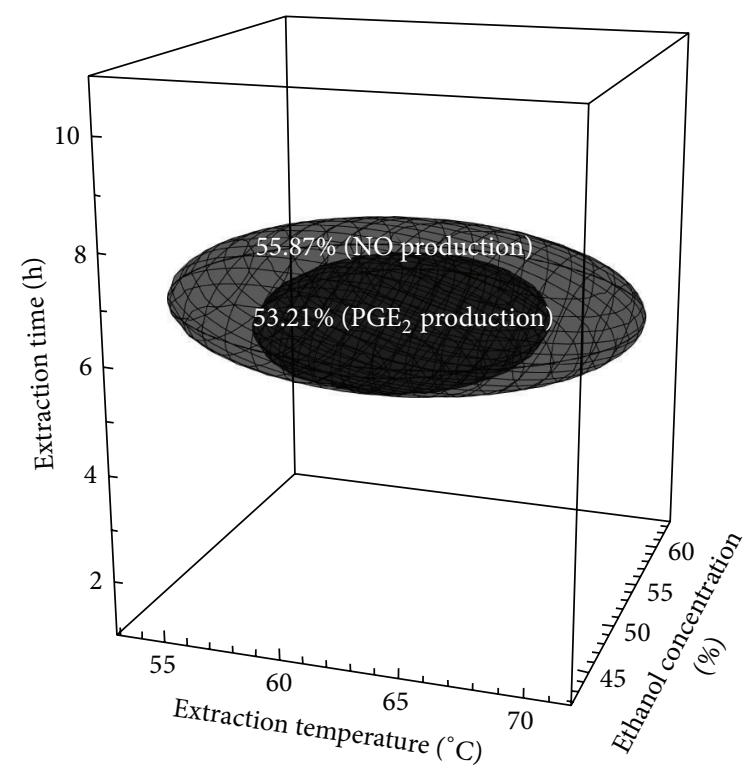

FIGURE 2: Superimposed response surface plots of the LPS-induced $\mathrm{PGE}_{2}$ and NO production showing optimal conditions for obtaining the extracts (Artemisia capillaris Thunb.).

suppression of NO production has been emphasized for the treatment of inflammation-related diseases [19]. During an inflammatory response, a proinflammatory gene such as inducible nitric oxide synthase (iNOS) is catalyzed through signal transduction pathways leading to $\mathrm{NO}$ production [37]. Lim et al. [31] reported that the ethanol extract of $A$. capillaris suppressed NO production via the downregulation of iNOS transcription. The mRNA and protein levels of iNOS were suppressed markedly by the ethanol extract of A. capillaris treatment in LPS-stimulated RAW 264.7 cells. Therefore, the ethanol extract of $A$. capillaris could be a good raw material for the development of drugs for the treatment of chronic inflammatory diseases [31]. These results demonstrate that the extraction conditions may contribute to the anti-inflammatory activity of $A$. capillaris in LPS-induced RAW 264.7 cells and may effectively yield a crude extract with high anti-inflammatory activity.

3.8. Prediction and Verification of Optimum Extraction Conditions. On the basis of the above findings, an optimization study was performed to evaluate the optimal extraction conditions for individual responses as well as the combination of all responses. First, the optimum ranges for extraction parameters of A. capillaris Thunb. were predicted by superimposing the 4-dimensional response surface plots of the LPS-induced $\mathrm{PGE}_{2}$ and NO production (Figure 2). The ranges of extraction conditions used for determination of the optimal conditions were extraction temperatures of $57-67^{\circ} \mathrm{C}$, ethanol concentrations of $46-60 \%$, and extraction times of $5.2-7.0 \mathrm{~h}$.

3.9. Cell Viability. Cell culture can be used to screen for toxicity both by estimation of the basal functions of the 
TABLE 3: Optimal extraction conditions determined by superimposing the response surfaces for extracts from Artemisia capillaris Thunb.

\begin{tabular}{lccrc}
\hline Independent variables & $\begin{array}{l}\text { Optional condition } \\
\text { (predicted ranges) }\end{array}$ & $Y_{1}{ }^{4}$ & $Y_{2}{ }^{5}$ & \multicolumn{2}{c}{ Experimental value } \\
\hline$X_{1}{ }^{2}$ & $61(57-65)$ & & & $Y_{2}$ \\
$X_{2}{ }^{2}$ & $51(45-57)$ & 53.87 & 56.16 & $52.65 \pm 1.01$ \\
$X_{3}{ }^{3}$ & $6.2(5.5-6.8)$ & & & $57.55 \pm 1.23$ \\
\hline
\end{tabular}

${ }^{1} X_{1}$ : extraction temperature $\left({ }^{\circ} \mathrm{C}\right)$

${ }^{2} X_{2}$ : ethanol concentration (\%)

${ }^{3} X_{3}$ : extraction time $(\mathrm{h})$.

${ }^{4} Y_{1}$ : LPS-induced $\mathrm{PGE}_{2}$ production (\%).

${ }^{5} Y_{2}$ : LPS-induced NO production (\%).

cell (i.e., processes common to all types of cells) or by tests on specialized cell functions [32]. Cell viability assays are used to identify the lack of certain toxic properties in the early stages of the development of potentially useful new substances such as therapeutic drugs, agricultural chemicals, and food additives. Therefore, a cytotoxicity test is a scientific analysis of the effects of toxic chemical substances on cultured mammalian cells. Cytotoxicity should be considered as a response variable in addition to LPS-induced $\mathrm{PGE}_{2}$ and NO production. The model adequacy of cytotoxicity was also predicted through regression analysis and ANOVA. The results of the ANOVA $R^{2}$ and adj. $R^{2}$ were 0.8941 and 0.7988 , respectively (Table 2 ). The model could be expressed as follows:

$$
\begin{aligned}
\text { Cytotoxicity }= & 72.885069+0.320093 X_{1} \\
& -0.037478 X_{2}+3.116604 X_{3} \\
& -0.004113 X_{1}{ }^{2}+0.002561 X_{2}^{2} \\
& -0.306064 X_{3}^{2}+0.002755 X_{1} X_{2} \\
& +0.002083 X_{1} X_{3}+0.017361 X_{2} X_{3} .
\end{aligned}
$$

The results of the canonical analysis revealed that the stationary point was the maximum and cytotoxicity showed the maximum predicted value at $91.1 \%$ at $56^{\circ} \mathrm{C}$ extraction temperature, $45 \%$ ethanol concentration, and $6.5 \mathrm{~h}$ extraction time.

Finally, the optimum ranges for the extraction parameters were predicted by superimposing the 4-dimensional response surface plots of LPS-induced $\mathrm{PGE}_{2}$ and $\mathrm{NO}$ production and by cytotoxicity of $A$. capillaris Thunb. extracts (Figure 3 ). The ranges of extraction conditions used for the determination of the optimal conditions were an extraction temperature of 57$65^{\circ} \mathrm{C}$, ethanol concentrations of $45-57 \%$, and an extraction time of 5.5-6.8 h. In order to validate the predicted optimal extraction conditions for both components, an optional midpoint for each condition was selected within the ranges, that is, extraction temperature of $61^{\circ} \mathrm{C}$, ethanol concentration of $51 \%$, and extraction time of $6.2 \mathrm{~h}$ (Table 3). The experimental results for the LPS-induced $\mathrm{PGE}_{2}$ and $\mathrm{NO}$ production were $52.65 \pm 1.01 \%$ and $57.55 \pm 1.23 \%$, respectively, and the results were in close agreement with the predicted values (LPS-induced $\mathrm{PGE}_{2}$ production: $53.87 \%$; LPS-induced NO production: $56.16 \%$ ) based on a response regression within

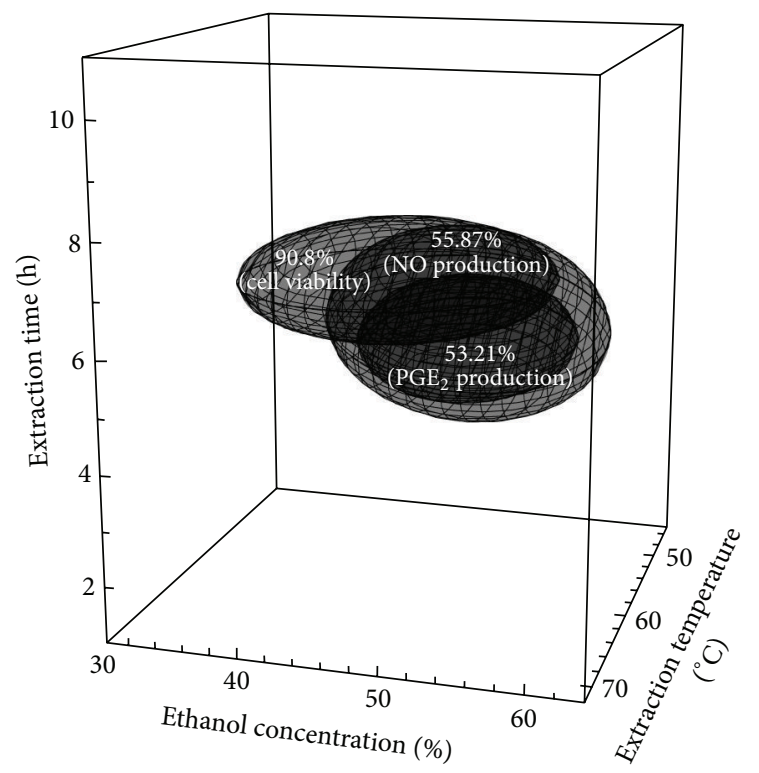

FIGURE 3: Superimposed response surface plots of cytotoxicity and LPS-induced $\mathrm{PGE}_{2}$ and $\mathrm{NO}$ production showing optimal conditions for obtaining the extracts (Artemisia capillaris Thunb.).

95\% confidence intervals of the experimental values. As a result, the model from a central composite design was considered to be accurate and reliable for predicting the reduction in the LPS-induced $\mathrm{PGE}_{2}$ and $\mathrm{NO}$ production of extracts at the cellular level.

3.10. HPLC Analysis. A typical HPLC chromatogram of phenolic and flavonoid compounds in the A. capillaris Thunb. extract at a point selected within the optimal ranges (extraction temperature, $62^{\circ} \mathrm{C}$; ethanol concentration, $53 \%$; extraction time, $6.1 \mathrm{~h}$ ) is presented in Figure 4 . The amount of selected phenolic and flavonoid compounds detected in the analyzed samples is shown in Table 4. Results are expressed in milligrams per $\mathrm{g}$ of dry sample.

\section{Conclusion}

This study indicates that the effects of extraction temperature, ethanol concentration, and extraction time on antiinflammatory activities were significant, and the predicted 


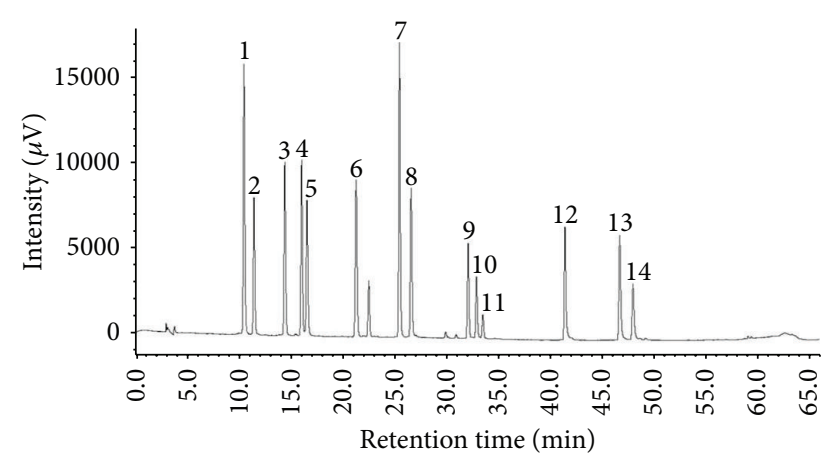

(a)

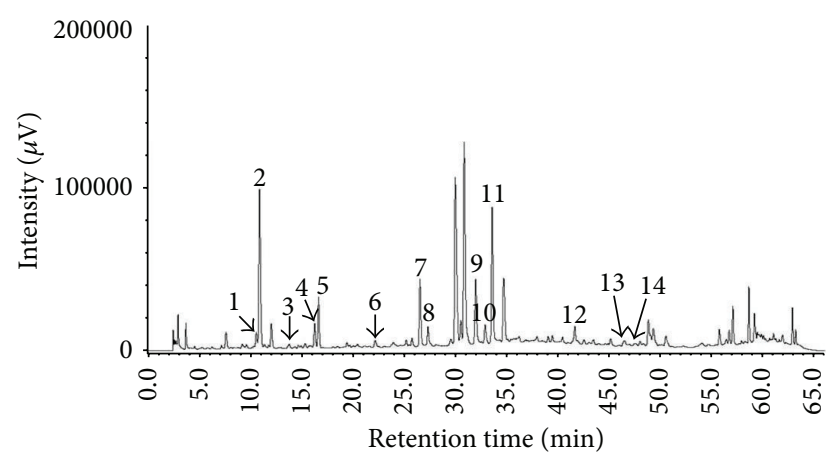

(b)

FIGURE 4: Typical HPLC chromatogram of phenolic and flavonoid (a) standards and (b) compounds in the Artemisia capillaris Thunb. extract at a point selected within the optimal ranges (extraction temperature, $62^{\circ} \mathrm{C}$; ethanol concentration, $53 \%$; extraction time, $6.1 \mathrm{~h}$ ). Peaks: 1 , chlorogenic acid; 2, catechin; 3, caffeic acid; 4, epicatechin; 5, epigallocatechin gallate; 6 , coumaric acid; 7, rutin; 8, catechin gallate; 9 , naringin; 10, apigenin-7-glucoside; 11, hesperidin; 12, quercetin; 13, apigenin; 14, kaempferol.

TABLE 4: Contents of selected phenolic and flavonoid compounds in the Artemisia capillaris Thunb. extract at a point selected within the optimal ranges (extraction temperature, $62^{\circ} \mathrm{C}$; ethanol concentration, 53\%; extraction time, $6.1 \mathrm{~h}$ ).

\begin{tabular}{lcc}
\hline & Compounds & Contents $\left(\mathrm{mg} \mathrm{g}^{-1}\right)^{1}$ \\
\hline \multirow{3}{*}{ Phenolic compounds } & Chlorogenic acid & $1.43 \pm 0.10$ \\
& Caffeic acid & $0.16 \pm 0.01$ \\
& Coumaric acid & $0.21 \pm 0.03$ \\
\hline & Rutin & $8.33 \pm 0.95$ \\
Flavonoids & Naringin & $6.69 \pm 0.70$ \\
& Apigenin-7-glucoside & $2.79 \pm 0.63$ \\
& Hesperidin & $57.44 \pm 7.14$ \\
& Quercetin & $1.98 \pm 0.12$ \\
& Apigenin & $3.13 \pm 0.53$ \\
Catechins & Kaempferol & $0.51 \pm 0.04$ \\
\hline & Catechin & $51.76 \pm 6.83$ \\
& Epicatechin & $2.60 \pm 0.52$ \\
& Epigallocatechin gallate & $12.29 \pm 1.60$ \\
& Catechin gallate & $2.61 \pm 0.48$ \\
\hline
\end{tabular}

${ }^{1}$ Values are mean \pm SD of triplicate determinations.

second-order polynomial models of the LPS-induced $\mathrm{PGE}_{2}$ and NO production and cytotoxicity in RAW 264.7 cells were also significant and suitable. The optimal conditions determined by superimposing the 4-dimensional response surface plots of all the responses $\left(Y_{1}, Y_{2}\right.$, and $\left.Y_{3}\right)$ were as follows: an extraction temperature of $57-65^{\circ} \mathrm{C}$, ethanol concentration of $45-57 \%$, and extraction time of 5.5-6.8 h. The present study is, to the best of our knowledge, the first to establish the optimal extraction conditions for improving the anti-inflammatory activity of $A$. capillaris Thunb. by utilizing the response variables from in vitro analysis and RSM.

\section{Conflict of Interests}

The authors declare that there is no conflict of interests regarding the publication of this paper.

\section{References}

[1] P. P. Joy, J. Thomas, M. Samuel, and B. P. Skaria, Medicinal Plants, Kerala Agricultural University Aromatic and Medicinal Plants Research Station, Kerala, India, 1998.

[2] Z. Amirghofran, "Herbal medicines for immunosuppression," Iranian Journal of Allergy, Asthma and Immunology, vol. 11, no. 2, pp. 111-119, 2012.

[3] J.-D. Cha, M.-R. Jeong, S.-I. Jeong et al., "Chemical composition and antimicrobial activity of the essential oils of Artemisia scoparia and A. capillaris," Planta Medica, vol. 71, no. 2, pp. 186190, 2005.

[4] E.-K. Kim, K.-B. Kwon, M.-J. Han et al., "Inhibitory effect of Artemisia capillaris extract on cytokine-induced nitric oxide formation and cytotoxicity of RINm5F cells," International Journal of Molecular Medicine, vol. 19, no. 3, pp. 535-540, 2007.

[5] J. H. Hong and I. S. Lee, "Cytoprotective effect of Artemisia capillaris fractions on oxidative stress-induced apoptosis in V79 cells," BioFactors, vol. 35, no. 4, pp. 380-388, 2009.

[6] Y. S. Kim, K. N. Bahn, C. K. Hah, H. I. Gang, and Y. L. Ha, "Inhibition of 7,12-dimethylbenz[a]anthracene induced mouse skin carcinogenesis by Artemisia capillaris," Journal of Food Science, vol. 73, no. 1, pp. T16-T20, 2008.

[7] A. Minagar, P. Shapshak, R. Fujimura, R. Ownby, M. Heyes, and C. Eisdorfer, "The role of macrophage/microglia and astrocytes in the pathogenesis of three neurologic disorders: HIVassociated dementia, Alzheimer disease, and multiple sclerosis," Journal of the Neurological Sciences, vol. 202, no. 1-2, pp. 13-23, 2002.

[8] F. Berenbaum, "Proinflammatory cytokines, prostaglandins, and the chondrocyte: mechanisms of intracellular activation," Joint Bone Spine, vol. 67, no. 6, pp. 561-564, 2000.

[9] J. H. J. Vernooy, M. A. Dentener, R. J. van Suylen, W. A. Buurman, and E. F. M. Wouters, "Long-term intratracheal lipopolysaccharide exposure in mice results in chronic lung inflammation and persistent pathology," American Journal of Respiratory Cell and Molecular Biology, vol. 26, no. 1, pp. 152159, 2002.

[10] T. Sakagami, J. Vella, M. F. Dixon et al., "The endotoxin of Helicobacter pylori is a modulator of host-dependent gastritis," Infection and Immunity, vol. 65, no. 8, pp. 3310-3316, 1997. 
[11] S. Fichtner-Feigl, I. J. Fuss, J. C. Preise, W. Strober, and A. Kitani, "Treatment of murine Th1- and Th2-mediated inflammatory bowel disease with NF- $\kappa$ B decoy oligonucleotides," Journal of Clinical Investigation, vol. 115, no. 11, pp. 3057-3071, 2005.

[12] L. Klotz, M. Schmidt, T. Giese et al., "Proinflammatory stimulation and pioglitazone treatment regulate peroxisome proliferator-activated receptor $\gamma$ levels in peripheral blood mononuclear cells from healthy controls and multiple sclerosis patients," The Journal of Immunology, vol. 175, no. 8, pp. 49484955, 2005.

[13] N. C. Walsh, T. N. Crotti, S. R. Goldring, and E. M. Gravallese, "Rheumatic diseases: the effects of inflammation on bone," Immunological Reviews, vol. 208, pp. 228-251, 2005.

[14] P. Arulpriya and P. Lalitha, "Evaluation of different extraction methods for optimization of extraction of aerial roots of Rhaphidophora aurea entwined over two diverse host trees," International Journal of ChemTech Research, vol. 5, no. 5, pp. 2173-2176, 2013.

[15] A. M. Gupta, M. Naraniwal, and V. Kothati, "Modern extraction methods for preparation of bioactive plant extracts," International Journal of Applied and Natural Sciences, vol. 1, no. 1, pp. 8-26, 2012.

[16] L. S. Lee, N. Lee, Y. H. Kim et al., "Optimization of ultrasonic extraction of phenolic antioxidants from green tea using response surface methodology," Molecules, vol. 18, no. 11, pp. 13530-13545, 2013.

[17] V. Kothari, A. Punjabi, and S. Gupta, "Optimization of microwave-assisted extraction of Annona squamosa seeds," ICFAI Journal of Life Sciences, vol. 3, no. 1, pp. 55-60, 2009.

[18] F. Denizot and R. Lang, "Rapid colorimetric assay for cell growth and survival-modifications to the tetrazolium dye procedure giving improved sensitivity and reliability," Journal of Immunological Methods, vol. 89, no. 2, pp. 271-277, 1986.

[19] A. C. Tinker and A. V. Wallace, "Selective inhibitors of inducible nitric oxide synthase: potential agents for the treatment of inflammatory diseases?" Current Topics in Medicinal Chemistry, vol. 6, no. 2, pp. 77-92, 2006.

[20] J. A. Williams and E. Shacter, "Regulation of macrophage cytokine production by prostaglandin $\mathrm{E}_{2}$. Distinct roles of cyclooxygenase-1 and -2," The Journal of Biological Chemistry, vol. 272, no. 41, pp. 25693-25699, 1997.

[21] J. Sun, X. Zhang, M. Broderick, and H. Fein, "Measurement of nitric oxide production in biological systems by using griess reaction assay," Sensors, vol. 3, no. 8, pp. 276-284, 2003.

[22] M. L. Abell and J. P. Braselton, The Mathematica Handbook, Compatible with Mathematica, Academic Press, Boston, Mass, USA, 1992.

[23] M. Nakanishi and D. W. Rosenberg, "Multifaceted roles of PGE2 in inflammation and cancer," Seminars in Immunopathology, vol. 35, no. 2, pp. 123-137, 2013.

[24] T. K. Trinh and S. K. Lim, "Application of response surface method as an experimental design to optimize coagulation tests," Environmental Engineering Research, vol. 15, no. 2, pp. 6370, 2010.

[25] B. Chauhan and R. Gupta, "Application of statistical experimental design for optimization of alkaline protease production from Bacillus sp. RGR-14," Process Biochemistry, vol. 39, no. 12, pp. 2115-2122, 2004.

[26] M. Sridevi and T. R. Genitha Er, "Optimization of osmotic dehydration process of pineapple by response surface methodology," Journal of Food Processing \& Technology, vol. 3, no. 8, article 173, pp. 1-7, 2012.
[27] A. C. Atkinson and A. N. Doney, Optimum Experimental Designs, Oxford University Press, Oxford, UK, 1992.

[28] S. Liu, F. Yang, C. Zhang, H. Ji, P. Hong, and C. Deng, "Optimization of process parameters for supercritical carbon dioxide extraction of Passiflora seed oil by response surface methodology," The Journal of Supercritical Fluids, vol. 48, no. 1, pp. 9-14, 2009.

[29] S. D. Lee, H. H. Park, D. W. Kim, and B. H. Bang, "Bioactive constituents and utilities of Artemisia sp. as medicinal herb and foodstuff," The Korean Journal of Food and Nutrition, vol. 13, no. 5, pp. 490-505, 2000.

[30] X. J. Wang, H. Sun, and Z. S. Liu, "Quantitative analysis of 6,7-dimethylesculetin and capillarisine in Artemisia capillaris Thunb. and prescriptions containing the crude drug," Zhongguo Zhong Yao Za Zhi, vol. 19, no. 11, pp. 667-702, 1994.

[31] H.-K. Lim, S. K. Cho, S. G. Park, and M. J. Cho, "Inhibitory effects of an ethanol extract of Artemisia capillaris on inflammatory mediators from LPS-stimulated RAW 264.7 cells," Journal of the Korean Society for Applied Biological Chemistry, vol. 53, no. 3, pp. 275-282, 2010.

[32] B. Ekwall, "Screening of toxic compounds in mammalian cell cultures," Annals of the New York Academy of Sciences, vol. 407, pp. 64-77, 1983.

[33] J. M. McCoy, J. R. Wicks, and L. P. Audoly, "The role of prostaglandin $\mathrm{E} 2$ receptors in the pathogenesis of rheumatoid arthritis," Journal of Clinical Investigation, vol. 110, no. 5, pp. 651658, 2002.

[34] T. G. Brock, R. W. McNish, P. Mancuso, M. J. Coffey, and M. Peters-Golden, "Prolonged lipopolysaccharide inhibits leukotriene synthesis in peritoneal macrophages: mediation by nitric oxide and prostaglandins," Prostaglandins and Other Lipid Mediators, vol. 71, no. 3-4, pp. 131-145, 2003.

[35] U. Panzer and M. Uguccioni, "Prostaglandin E2 modulates the functional responsiveness of human monocytes to chemokines," European Journal of Immunology, vol. 34, no. 12, pp. 3682-3689, 2004.

[36] I. Istadi and N. A. S. Amin, "Optimization of process parameters and catalyst compositions in carbon dioxide oxidative coupling of methane over $\mathrm{CaO}-\mathrm{MnO} / \mathrm{CeO}_{2}$ catalyst using response surface methodology," Fuel Processing Technology, vol. 87, no. 5, pp. 449-459, 2006.

[37] M.-H. Pan, C.-S. Lai, S. Dushenkov, and C.-T. Ho, "Modulation of inflammatory genes by natural dietary bioactive compounds," Journal of Agricultural and Food Chemistry, vol. 57, no. 11, pp. 4467-4477, 2009. 

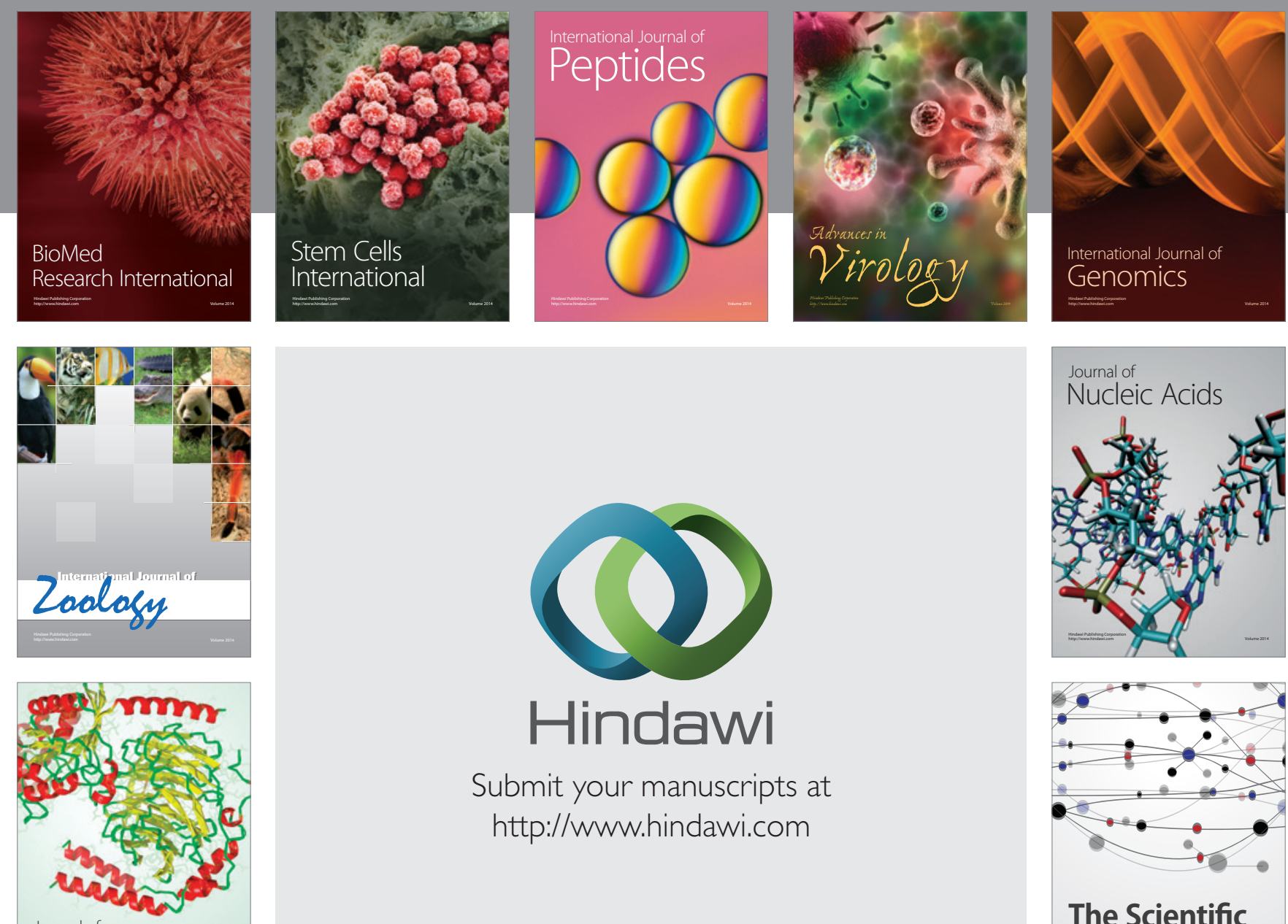

Submit your manuscripts at

http://www.hindawi.com

Journal of
Signal Transduction
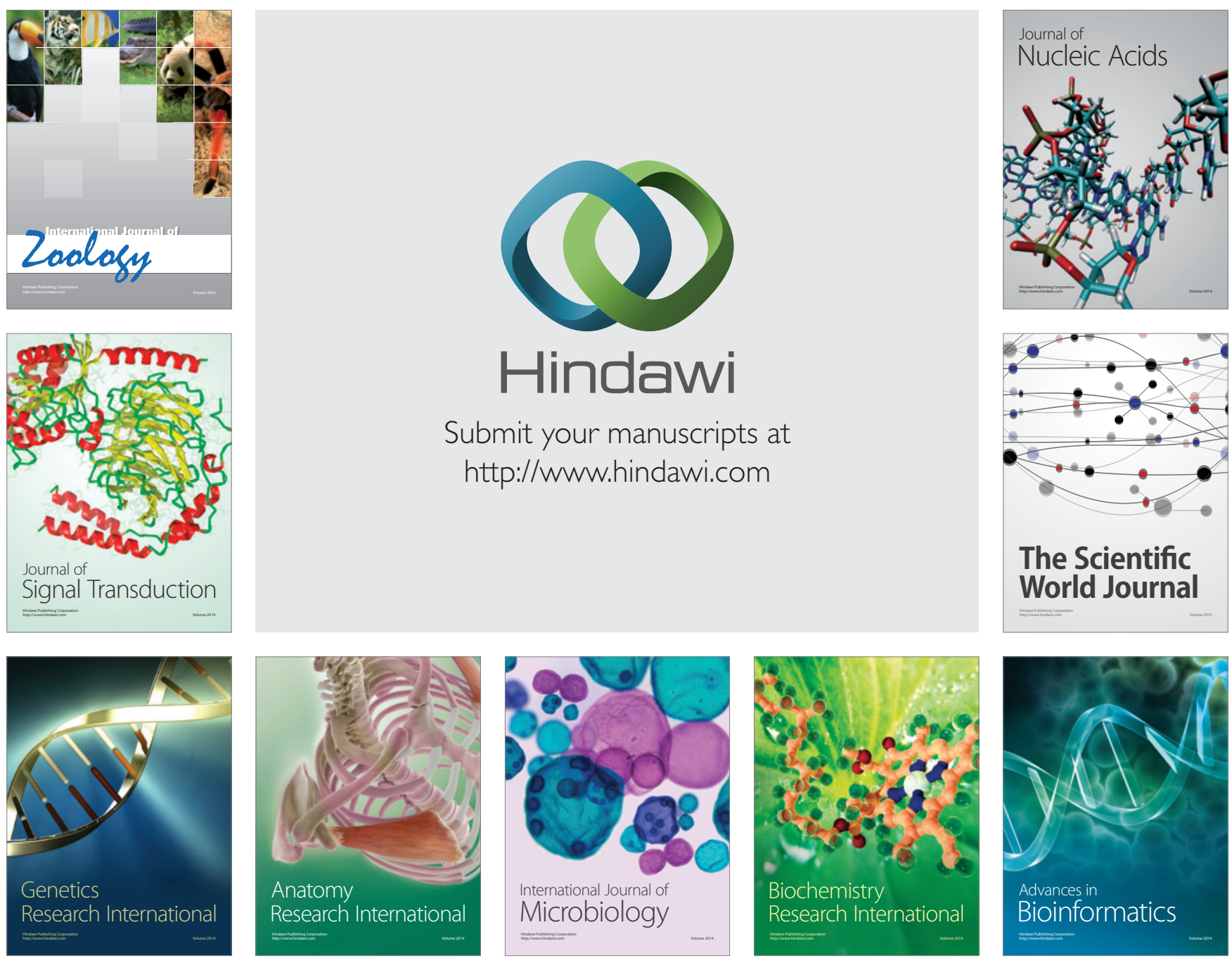

The Scientific World Journal
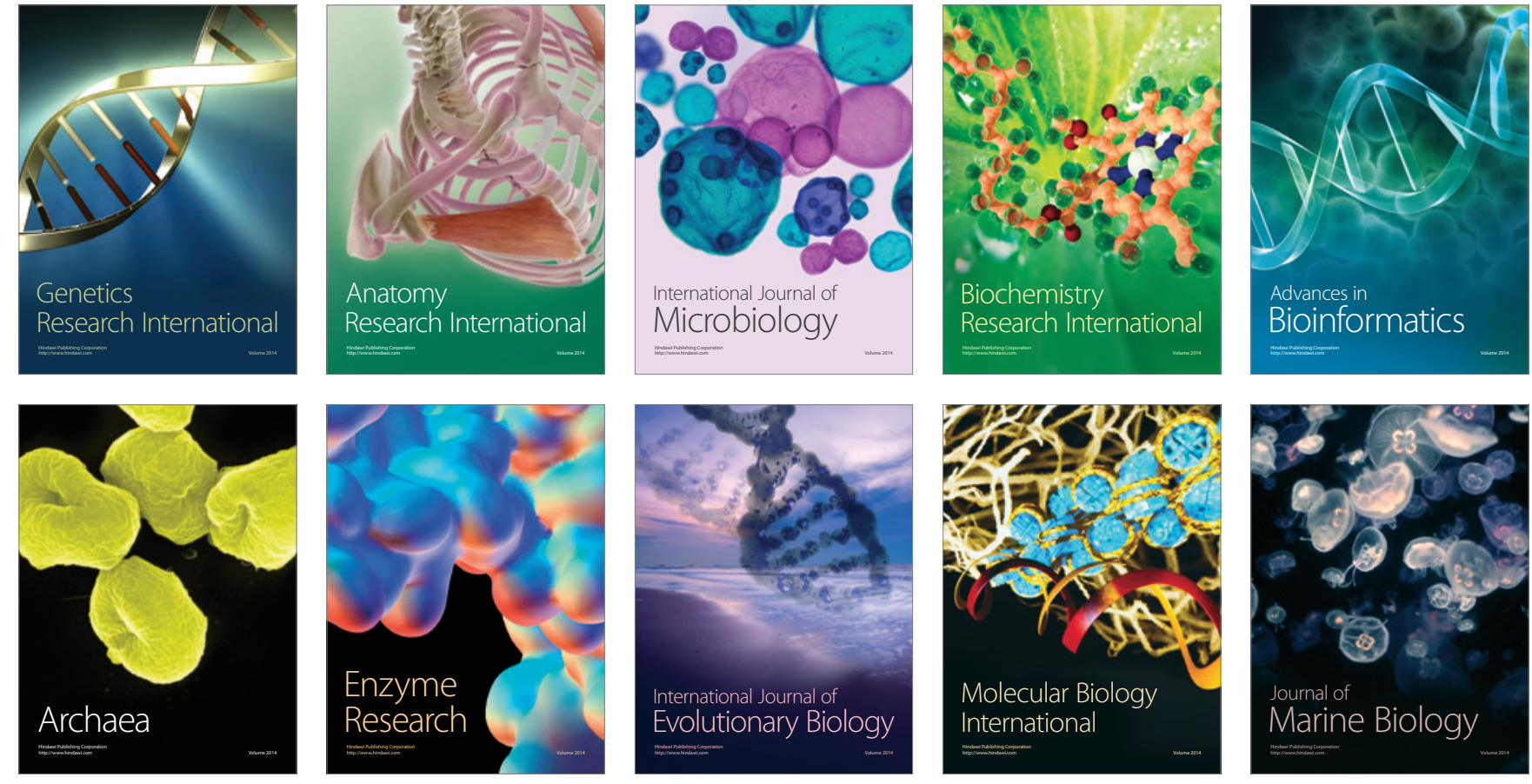